\title{
Supragingival biofilm control and systemic inflammation in patients with type 2 diabetes mellitus
}

\begin{abstract}
Hilana Paula Carillo ARTESE(a) Priscila Larcher LONGO(b) Giovane Hisse GOMES(a) Marcia Pinto Alves MAYER(b) Giuseppe Alexandre ROMITO(a)
\end{abstract}

(a) Universidade de São Paulo - USP, School of Dentistry, Department of Periodontics, São Paulo, SP, Brazil.

(b) Universidade de São Paulo - USP, Institute of Biomedical Sciences, Department of Microbiology, São Paulo, SP, Brazil.

Declaration of Interests: The authors certify that they have no commercial or associative interest that represents a conflict of interest in connection with the manuscript.

Corresponding Author:

Giuseppe Alexandre Romito

E-mail: garomito@usp.br

DOI: 10.1590/1807-3107BOR-2015.vol29.0071

Submitted: Sep 23, 2014

Accepted for publication: Jan 28, 2015

Last revision: May 08, 2015
Abstract: The objective of this study was to evaluate the effect of strict supragingival biofilm control on serum inflammatory markers and on periodontal clinical parameters in type 2 diabetes mellitus (T2DM) patients with chronic severe periodontitis. Twenty-four individuals with T2DM and periodontitis were randomly allocated to two treatment groups. The supragingival therapy group $(\mathrm{ST}, \mathrm{n}=12)$ received supragingival scaling, whereas the intensive therapy group (IT, $\mathrm{n}=12$ ) underwent supra- and subgingival scaling, as well as root planing. Patients from both groups received professional oral hygiene instructions every month. Data regarding visible plaque index (VPI), gingival bleeding index (GBI), bleeding on probing (BOP), probing pocket depth (PPD), clinical attachment level (CAL), serum levels of interleukin (IL)-6, IL-17A, IL-8, tumor necrosis factor a (TNF-a), monocyte chemoattractant protein (MCP)-1 enzyme-linked immunosorbent assay (ELISA), and glycated hemoglobin (HbA1c) levels were obtained at baseline and at 6 months post-therapy. Both therapies resulted in the improvement of almost all clinical periodontal parameters $(p<0.05)$. There were no differences in TNF- $\alpha$, IL-8, IL-17A and HbA1c levels in either group ( $p>0.05)$, between the two periods. However, MCP-1 levels were significantly reduced in both the ST $(\mathrm{p}=0.034)$ and the IT $(\mathrm{p}=0.016)$ groups, whereas the serum IL-6 levels were significantly reduced only in the IT group $(p=0.001)$. Strict control of supragingival biofilm has a limited effect on systemic inflammatory markers, and a moderate effect on periodontal clinical parameters.

Keywords: Diabetes Mellitus; Periodontitis; Cytokines.

\section{Introduction}

Periodontitis is a destructive form of periodontal disease (PD), affecting approximately $50 \%$ of all adults and more than $60 \%$ of over 65-year-olds. Severe periodontitis is known to affect approximately $10 \%-15 \%$ of the population. ${ }^{1,2}$ Periodontal destruction is mediated by the host's immune-inflammatory response ${ }^{3}$ elicited by pathogendominated biofilms. This response leads to the production of specific antibodies and inflammatory mediators within the local tissues, as well as a systemically elicited response. ${ }^{4}$ 
Cytokines participate actively in the initiation and maintenance of immune responses to oral bacteria. ${ }^{5}$ An imbalance between pro- and anti-inflammatory cytokines leads to the destruction of periodontal tissue and to clinical signs of periodontal disease. ${ }^{6}$ The local production of cytokines in response to periodontal bacteria is related to serum concentrations of pro-inflammatory cytokines. ${ }^{7}$ Non-surgical periodontal therapy promotes the reduction of bacterial challenge, ${ }^{8,9,10}$ and may also affect the inflammatory burden. ${ }^{11}$

Diabetes and PD are highly prevalent in the population, and share a common etiopathogenesis. ${ }^{12}$ Type 2 diabetes mellitus (T2DM) is a recognized risk factor for PD, affecting its prevalence, incidence, and severity; ${ }^{13,14}$ the relationship between the two disorders is bidirectional ${ }^{12}$. PD may represent an additional factor contributing to the total inflammatory burden in individuals with T2DM. Furthermore, periodontal treatment has been shown to improve metabolic control in T2DM. ${ }^{15,16}$

The role of periodontal treatment in serum inflammatory markers is somewhat conflicting, possibly because of differences in periodontal therapy protocols (time of follow-up and use of local/systemic antimicrobials). Some studies have shown considerable changes in the serum levels of IL-6 and TNF- $\alpha,{ }^{17,18}$ whereas others have disclosed no significant changes in TNF- $a,{ }^{19}$ IL- $6,{ }^{20}$ and IL-8. ${ }^{21}$ To the best of our knowledge, there is no information regarding the effect of strict supragingival biofilm control on inflammatory burden. Thus, the purpose of this study was to test the null hypothesis that supragingival scaling does not have beneficial effects on the serum levels of inflammatory markers or on periodontal clinical parameters in patients with chronic generalized periodontitis and T2DM, compared with the effects of intensive periodontal therapy (supra- and subgingival scaling, as well as root planing).

\section{Methodology Study population}

Patients selected for the study were recruited between February 2011 and December 2013 in complete accordance with the Helsinki Declaration of 1975, as revised in 2000. Ethical approval was received by the Ethics Committee of the Universidade de São Paulo SP, São Paulo, Brazil (FR 284313-127/2009 protocol). All patients with T2DM were diagnosed according to the World Health Organization classification, ${ }^{22}$ and remained under the supervision of an endocrinologist. The participants were treated with a dietary intervention and/or oral hypoglycemic agents.

The inclusion criteria were as follows: individuals $\geq 35$ years of age, confirmed diagnosis of T2DM for a period of over 3 years, generalized severe chronic periodontitis (number of probing pocket depth [PPD] sites $\geq 30 \%$, clinical attachment level [CAL] $>4 \mathrm{~mm}$, and bleeding on probing), and $\geq 15$ teeth. Pregnant women, smokers, patients with body mass index (BMI) $>35 \mathrm{~kg} / \mathrm{m}^{2}$, or those who had received periodontal therapy, systemic antibiotic, or oral antiseptic therapy 6 months prior to the study were excluded.

Sample size calculation was undertaken by assuming a reduction of $2 \mathrm{~mm}$ in mean pocket depth, with $0.6 \mathrm{~mm}$ standard deviation in the IT group, and $1 \mathrm{~mm}$ mean pocket depth reduction, with $0.6 \mathrm{~mm}$ standard deviation in the ST group ( $90 \%$ statistical power and $5 \%$ significance level). The required sample size for each group was determined as 11 , and 12 patients were recruited to account for potential dropouts and missing data.

\section{Periodontal Clinical Data}

The presence of supragingival biofilm was recorded as visible plaque index (VPI) ${ }^{23}$ whereas marginal gingival bleeding was recorded as gingival bleeding index (GBI). ${ }^{23}$ Bleeding on probing (BOP), PPD, and CAL were also evaluated. The North Carolina manual probe was used in this study, (Hu-Friedy ${ }^{\circledR}$, Chicago, USA); VPI, GBI, and BOP were recorded as (0) absent, or (1) present. Clinical examinations were performed by two blinded and calibrated examiners (H.P.C.A. and A.M. F.) on six sites per tooth (excluding the third molars), at baseline and at 6 months after periodontal therapy. Reproducibility during the study was assessed in $10 \%$ of the participants, with intraclass correlation coefficients ranging from 0.90 to 0.94 for periodontal PPD, and 0.85 to 0.89 for CAL. 


\section{Experimental Design and Periodontal Therapy}

This study comprised 24 individuals randomly assigned by a computer random number generator (Excel), and allocated by sequentially numbered sealed opaque envelopes. All individuals were given oral hygiene instructions. The supragingival therapy group (ST, $n=12$ ) received supragingival scaling using an ultrasonic device and periodontal curettes (Hu-Friedy ${ }^{\circledR}$, Chicago, USA). A Single appointment lasted $\sim 60$ minutes. The intensive therapy group (IT, $\mathrm{n}=12$ ) received supra- and subgingival scaling and root planing, (in sites with PPD $\geq 4 \mathrm{~mm}$ ) using an ultrasonic device and periodontal curettes. The procedures for the IT group were performed under local anesthesia ( $3 \%$ prilocaine with felypressin), in two appointments lasting 120 minutes each. Supportive therapy for biofilm control consisted of professional instructions on oral hygiene given to each patient every month. Periodontal therapy was carried out by an experienced periodontist (G.H.G.). No changes in medication or diet were made during the study period.

\section{Inflammatory Markers and HbAlc Measurements}

Samples of approximately $5 \mathrm{~mL}$ of blood were collected by venipuncture and placed in untreated Vacutainer blood collection tubes (Becton Dickinson Co., São Paulo, Brazil). An experienced nurse technician (J.P.R.) performed the blood collections. Glycated hemoglobin (HbA1c) was analyzed by high-performance liquid chromatography (DiaSTAT Hemoglobin A1c Analyzer System, BioRad Laboratories, Hercules, USA), at the clinical laboratory located in the Hospital Universitário - HU (University Hospital), at the USP. The serum was obtained by centrifugation at 2,000 $\mathrm{g}$ for 10 minutes, aliquoted, and stored at $-80^{\circ} \mathrm{C}$ until further analysis.

Quantitative measurements of IL-6, IL-8, IL-17, TNF- $\alpha$ and MCP-1 were assayed by enzyme-linked immunosorbent assay (ELISA) (Peprotech Inc., Rockyhill, USA), according to the manufacturer's instructions, whereas the optical density was determined using a Micro Plate reader model 680 (BioRad Laboratories Inc., Hercules, USA). Serum from each patient was tested in triplicates.

\section{Statistical analysis}

Data from the ELISA were analyzed using Prism software (Prism 5 Project, Graphpad Software Inc., La Jolla, USA). Shapiro-Wilk normality test was used and the data did not follow Gaussian distribution. The data were analyzed by non-parametric statistical methods. Periodontal clinical data were analyzed using SPSS software (SPSS for Windows, version 17.0, SPSS Inc., Chicago, USA), considering the individual as a study unit. Intergroup comparison was determined using the Mann-Whitney test. The intragroup comparison was determined using the Wilcoxon signed rank test. The tests were based on the median values with variability measures ( $25 \%$ and $75 \%$ quartiles). The results were considered statistically significant at $\mathrm{p}<0.05$. The GBI, VPI, and BOP data were obtained by calculating percentages of positive sites per patient, and thereafter, median values were calculated for the groups. For PPD and CAL, measured in millimeters, the median value per patient was obtained first, followed by the median value for the group.

\section{Results}

All patients selected for analysis in the present study completed 6 months of the clinical trial. The ST group was comprised of $56.3 \%$ women (mean age, $54.4 \pm 5.8$ years; BMI, $25.6 \pm 4.4$ ), whereas the IT group was comprised of $52.0 \%$ women (mean age, $52.0 \pm 3.3$ years; BMI, $26.9 \pm 3.8$ ). Both groups were similar in age, gender, and BMI at baseline $(p>0.05)$.

No significant differences in periodontal parameters were observed between the two groups at baseline (Table 1). Both therapies resulted in statistically significant improvements in most clinical parameters after 6 months of follow-up, except for CAL in the ST group. However, the effects on BOP and PPD were more pronounced in the IT group, with a significant gain in CAL observed in this group only (Table 1). Changes in periodontal clinical parameters are represented in Table 2. The effectiveness of periodontal treatment on PPD (4-6 mm; $\geq 7 \mathrm{~mm}$ ) and CAL (4-6 $\mathrm{mm} ; \geq 7 \mathrm{~mm}$ ) was observed in both groups. Changes in PPD $(4-6 \mathrm{~mm})(\mathrm{p}=0.001)$ and CAL $(4-6 \mathrm{~mm})(\mathrm{p}=0.001)$ were found to be significantly different between the two groups in this study (Table 2). 
Table 1. Medians values $\left(75^{\text {th }} / 25^{\text {th }}\right.$ percentiles $)$ for clinical periodontal parameters at baseline and 6 months post-therapy for the supragingival therapy (ST; $n=12$ ) and the intensive periodontal therapy (IT; $n=12)$ groups.

\begin{tabular}{|c|c|c|c|}
\hline \multirow{2}{*}{ Clinical parameters } & \multicolumn{2}{|c|}{ Groups } & \multirow{2}{*}{$\mathrm{p}$-value } \\
\hline & $\mathrm{ST}(\mathrm{n}=12)$ & $\mathrm{IT}(\mathrm{n}=12)$ & \\
\hline \multicolumn{4}{|l|}{ VPI (\%) } \\
\hline Baseline & $87.80(63.60-97.02)$ & 72.60 (59.90-97.27) & 0.57 \\
\hline 6 months & $43.20(12.50-72.60)$ & $26.60(7.42-33.60)$ & 0.25 \\
\hline$p$-value & $0.03^{*}$ & $0.005^{*}$ & \\
\hline \multicolumn{4}{|l|}{ GBI (\%) } \\
\hline Baseline & $56.30(40.00-81.20)$ & 68.20 (31.75-88.55) & 0.15 \\
\hline 6 months & $17.30(13.20-49.60)$ & $12.20(4.20-20.27)$ & 0.12 \\
\hline$p$-value & $0.004^{*}$ & $0.007^{*}$ & \\
\hline \multicolumn{4}{|l|}{ PPD (mm) } \\
\hline Baseline & $4.47(3.03-4.66)$ & $4.75(3.12-4.96)$ & 0.10 \\
\hline 6 months & 3.30 (2.80-3.375) & $2.32(2.05-2.78)$ & $0.01^{\#}$ \\
\hline$p$-value & $0.04^{*}$ & $0.005^{*}$ & \\
\hline \multicolumn{4}{|l|}{$\mathrm{CAL}(\mathrm{mm})$} \\
\hline Baseline & $4.77(3.94-5.64)$ & $4.29(3.67-5.13)$ & 0.07 \\
\hline 6 months & $4.26(3.46-4.99)$ & $3.31(2.94-4.18)$ & $0.05^{\#}$ \\
\hline$p$-value & 0.06 & $0.006^{*}$ & \\
\hline \multicolumn{4}{|l|}{$\mathrm{BOP}(\%)$} \\
\hline Baseline & 73.20 (55.40-83.90) & $60.70(47.40-69.07)$ & 0.06 \\
\hline 6 months & $42.09(23.80-49.40)$ & $10.70(6.07-20.20)$ & $0.001^{\#}$ \\
\hline $\mathrm{p}$-value & $0.03^{*}$ & $0.005^{*}$ & \\
\hline
\end{tabular}

* $p$-values represent the intragroup (vertical) comparison between baseline and 6-month period (Wilcoxon's test; $p<0.05)$. " $p$-values represent the intergroup (horizontal) comparison between baseline and 6-month period (Mann-Whitney U-test; $\mathrm{p}<0.05$ ). VPI: visible plaque index;

$\mathrm{GBI}$ : gingival bleeding index; PPD: probing pocket depth; CAL: clinical attachment level; BOP: bleeding on probing.

Table 2. Changes in the periodontal clinical parameters PPD (4-6 mm; $\geq 7 \mathrm{~mm})$ and CAL (4-6 mm; $\geq 7 \mathrm{~mm})$ from baseline to 6 months post-therapy.

\begin{tabular}{|c|c|c|c|}
\hline \multirow{2}{*}{ Clinical parameters } & \multicolumn{3}{|c|}{ Mean Reduction (SEM) [95\%Cl] } \\
\hline & $\mathrm{ST}(\mathrm{n}=12)$ & $\mathrm{IT}(\mathrm{n}=12)$ & $p$-value \\
\hline PPD 4-6 mm (\%) & $10.1(2.9)[4.8-14.10]$ & \multicolumn{2}{|c|}{$23.3(2.0)[13.5-41.6] 0.001^{*}$} \\
\hline $\mathrm{PPD} \geq 7 \mathrm{~mm}(\%)^{£}$ & $3.8(0.9)$ [1.2-9.5] & \multicolumn{2}{|c|}{5.8 (1.2) [0.6-1 1.9] 0.298} \\
\hline CAL 4-6 mm (\%) $)^{\varepsilon}$ & $7.2(1.6)[1.2-16.7]$ & \multicolumn{2}{|c|}{14.6 (3.1) [3.0-33.9] $0.001^{*}$} \\
\hline $\mathrm{CAL} \geq 7 \mathrm{~mm}(\%)^{£}$ & $3.2(1.4)[0.8-14.1]$ & \multicolumn{2}{|c|}{4.8 (1.1) [0.6-15.5] 0.09} \\
\hline
\end{tabular}

¿Significant reductions in all periodontal parameters from baseline to 6-month period (Wilcoxon signed rank test; $p<0.05$ ).

*Significant difference between groups (Mann-Whitney test; $p$ < 0.05). PPD: Probing pocket depth; CAL: clinical attachment level.

The levels of inflammatory markers and HbA1c, at baseline and at the end of the experimental period, are shown in Figure 1. Both groups of patients showed similar levels of serum inflammatory markers at baseline $(p>0.05)$. No significant differences for serum levels of IL-8, IL-17A and TNF-a were recorded between the two groups ( $p>0.05), 6$ months after periodontal therapy.
However, there was a statistically significant reduction in IL-6 serum levels in the IT group, after 6 months of therapy ( $<0.001)$, but not in the ST group ( $\mathrm{p}=0.33$ ). Furthermore, MCP-1 levels were reduced in both groups (ST, $p=0.03 ; \mathrm{IT}, \mathrm{p}=0.01)$. There were no significant differences in serum HbA1c levels between the groups at baseline and at 6 months post-therapy $(p>0.05)$ (Figure 1). 

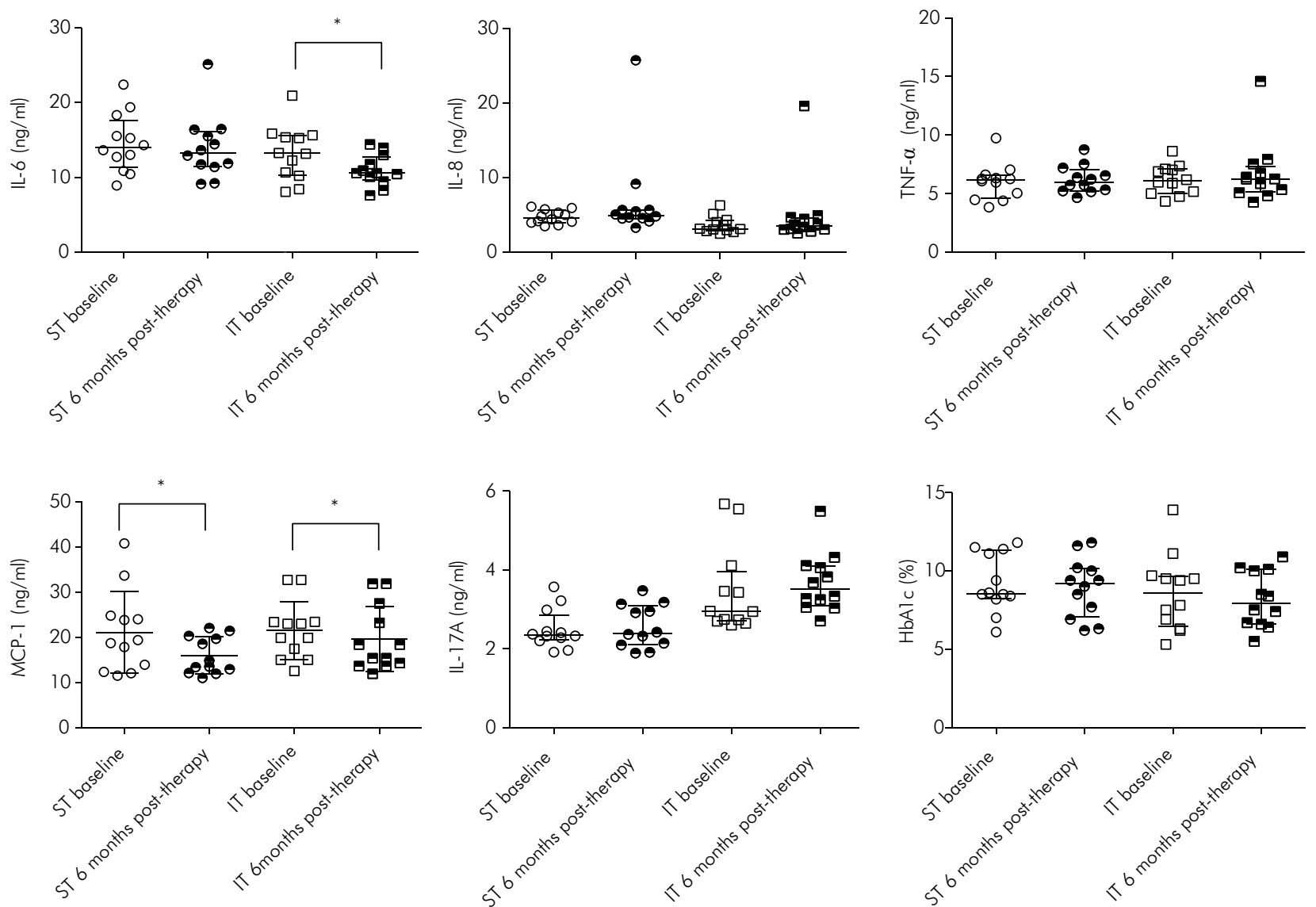

The diagram represents median values: inferior quartile (25\%) and superior quartile (75\%). ${ }^{*}$ Statistical difference between the two groups (ST and IT) at baseline and 6 months post-therapy ( $<0.05$; Wilcoxon signed rank test).

Figure 1. Distribution according to the serum levels of IL-6, MCP- I, IL-8, IL-17, TNF- $a$, and HbAlc at baseline and 6 months post-therapy in the supragingival therapy (ST) and intensive periodontal therapy (IT) groups.

\section{Discussion}

The results of the current study demonstrate that supragingival biofilm control reduces the local symptoms of inflammation, with a limited systemic effect on serum biomarkers in T2DM individuals. This is in concurrence with the findings from previous clinical studies that have reported similar effects of periodontal therapy on IL-6 levels. ${ }^{17,24}$ To our knowledge, there are no previously published studies available regarding the effect of strict supragingival biofilm control on serum levels of IL-6, IL-8, IL-17, TNF- $\alpha$, and MCP-1 in T2DM patients. Our follow-up data showed that both therapies were comparable in promoting clinical improvement of the periodontal parameters; however, the best effect was seen in the IT group.

Minimal periodontal treatment in the form of supragingival scaling, prophylaxis, and oral hygiene instructions was given to the patients in the ST group. Although not considered the ideal form of therapy, it promoted clinical improvement of most of the periodontal parameters in this study. It has been known for decades that professional supragingival biofilm control has an effect on the subgingival microbiota, and, consequently, on periodontal clinical parameters. ${ }^{25}$ This effect is attributed to the reduced inflammation of adjacent periodontal tissues, leading to a restriction in the availability of nutrients necessary for bacterial multiplication. It is important to highlight that previous studies have not been performed on diabetic individuals. Moreover, in the present study, no microbiological or local cytokine level analyses were performed, and thereby are recommended for further studies on T2DM individuals.

In order to determine more specific differences between the two groups, periodontal sites were divided 
into two categories; intermediate (4-6 mm) and deep $(\geq 7 \mathrm{~mm})$, for both PPD and CAL parameters. The shallow sites $(\leq 3 \mathrm{~mm})$ revealed no considerable changes in PPD and CAL after treatment (data not shown). Clinical attachment gain was observed during analysis of the median values, and was significantly different in the IT group only (Table 2). Stratification and analysis of the changes in CAL $(4-6 ; \geq 7 \mathrm{~mm})$ revealed a gain in clinical attachment in both groups, with additional benefits observed in the IT group, indicating the importance of root debridement in treating diseased sites. We could speculate that the reduction of inflammation in the adjacent periodontal tissue was the major cause for the changes in PPD and CAL measurements.

The mechanisms by which periodontitis influences T2DM have not been precisely defined. Increasing evidence suggests that severe chronic periodontitis induces subclinical bacteremia, leading to a low-grade systemic inflammation that may affect metabolic control. ${ }^{26,27}$ Some of the inflammatory markers linked to T2DM complications and insulin resistance are IL-6, IL-12, MCP-1, and IL-8. Moreover, IL-6 has also been associated with vascular complications, ${ }^{28}$ and atherogenesis may be regulated by chemotactic factors, such as MCP-1, which has been linked to both insulin

\section{References}

1. Marcenes W, Kassebaum NJ, Bernabé E, Flaxman A, Naghavi M, Lopez A, et al. Global burden of oral conditions in 1990-2010: a systematic analysis. J Dent Res. 2013 Jul;92(7):592-7.

2. Chapple IL, Genco R, Working group 2 of the joint EFP/AAP workshop. Diabetes and periodontal diseases: consensus report of the Joint EFP/AAP Workshop on Periodontitis and Systemic Diseases. J Periodontol. 2013 Apr;84(4 Suppl):S106-12.

3. Salvi GE, Lang NP. Host response modulation in the management of periodontal diseases. J Clin Periodontol. 2005;32(Suppl 6):108-29.

4. Ebersole JL, Dawson DR 3rd, Morford LA, Peyyala R, Miller CS, Gonzaléz OA. Periodontal disease immunology: 'double indemnity' in protecting the host. Periodontol 2000. 2013 Jun;62(1):163-202.

5. Shin J, Kho SA, Choi YS, Kim YC, Rhyu IC, Choi Y. Antibody and $\mathrm{T}$ cell responses to Fusobacterium nucleatum and Treponema denticola in health and chronic periodontitis. PLoS One. 2013;8(1):e53703.

6. Preshaw PM, Taylor JJ. How has research into cytokine interactions and their role in driving immune responses resistance and hyperglycemia. Our study demonstrated that both therapies were able to reduce serum MCP-1 levels, as also achieved by some recently developed drugs. ${ }^{29}$ This reduction may be partly explained by the partial improvement of periodontal parameters and local inflammation. On the other hand, this partial periodontal improvement was not able to reduce IL-6 serum levels in the ST group.

\section{Conclusions}

Supragingival therapy has a modest effect on periodontal clinical parameters, and a limited effect on systemic inflammatory markers, compared with the effect of intensive periodontal therapy. Therefore, intensive periodontal therapy should be prioritized to control the inflammatory burden on T2DM individuals.

\section{Acknowledgments}

We would like to thank Dr. Adriana Moura Foz, Dr. Anna Carolina R. T. Horliana, Dr. Mariana de Sousa Rabelo and João Paulo Ribeiro, for their collaboration in the clinical procedures. This study was supported by the Fundação de Amparo à Pesquisa do Estado de São Paulo - FAPESP, São Paulo, Brazil, under protocol numbers 2011/06982-4;10057-4;18618-5.

impacted our understanding of periodontitis?. J Clin Periodontol. 2011 Mar;38(Suppl 11):60-84.

7. Górska R, Gregorek H, Kowalski J, Laskus-Perendyk A, Syczewska M, Madaliński K. Relationship between clinical parameters and cytokine profiles in inflamed gingival tissue and serum samples from patients with chronic periodontitis. J Clin Periodontol. 2003 Dec;30(12):1046-52.

8. Hellström MK, Ramberg P, Krok L, Lindhe J. The effect of supragingival plaque control on the subgingival microflora in human periodontitis. J Clin Periodontol. 1996 Oct;23(10):934-40.

9. Haffajee AD, Arguello EI, Ximenez-Fyvie LA, Socransky SS. Controlling the plaque biofilm. Int Dent J. 2003;53(Suppl 3):191-9.

10. Colombo AP, Teles RP, Torres MC, Rosalém W, Mendes MC, Souto RM, et al. Effects of non-surgical mechanical therapy on the subgingival microbiota of Brazilians with untreated chronic periodontitis: 9-month results. J Periodontol. 2005 May;76(5):778-84. 
11. Chen L, Wei B, Li J, Liu F, Xuan D, Xie B, et al. Association of periodontal parameters with metabolic level and systemic inflammatory markers in patients with type 2 diabetes. J Periodontol. 2010 Mar;81(3):364-71.

12. Bascones-Martínez A, González-Febles J, Sanz-Esporrín J. Diabetes and periodontal disease. Review of the literature. Am J Dent. 2014 Apr;27(2):63-7.

13. Seppälä B, Seppälä M, Ainamo JA. A longitudinal study on insulin-dependent diabetes mellitus and periodontal disease. J Clin Periodontol. 1993 Mar;20(3):161-5.

14. Soskolne WA, Klinger A. The relationship between periodontal disease and diabetes: an overview. Ann Periodontol. 2001 Dec;6(1):91-8.

15. Sgolastra F, Severino M, Pietropaoli D, Gatto R, Monaco A. Effectiveness of periodontal treatment to improve metabolic control in patients with chronic periodontitis and type 2 diabetes: a meta-analysis of randomized clinical trials. J Periodontol. 2013 Jul;84(7):958-73.

16. Sun WL, Chen LL, Zhang SZ, Wu YM, Ren YZ, Quin GM. Inflammatory cytokines, adiponectin, insulin resistance and metabolic control after periodontal intervention in patients with type 2 diabetes and chronic periodontitis. Intern Med. 2011;50(15):1569-74.

17. O'Connell PA, Taba M, Nomizo A, Foss Freitas MC, Suaid FA, Uyemura SA, et al. Effects of periodontal therapy on glycemic control and inflammatory markers. J Periodontol. 2008 May;79(5):774-83.

18. Dağ A, Firat ET, Arikan S, Kadiroğlu AK, Kaplan A. The effect of periodontal therapy on serum TNF-alpha and HbA1c levels in type 2 diabetic patients. Aust Dent J. 2009 Mar;54(1):17-22.

19. Chen L, Luo G, Xuan D, Wei B, Liu J, Zhang J. Effects of nonsurgical periodontal treatment on clinical response, serum inflammatory parameters, and metabolic control in patients with type 2 diabetes: a randomized study. J Periodontol. 2012 Apr;83(4):435-43.

20. Lin SJ, Tu YK, Tsai SC, Lai SM, Lu HK. Non-surgical periodontal therapy with and without subgingival minocycline administration in patients with poorly controlled type II diabetes: a randomized controlled clinical trial. Clin Oral Investig. 2012 Apr;16(2):599-609.

21. Correa FO, Gonçalves D, Figueredo CM, Bastos AS, Gustafsson A, Orrico SR. Effect of periodontal treatment on metabolic control, systemic inflammation and cytokines in patients with type 2 diabetes. J Clin Periodontol. 2010 Jan;37(1):53-8.

22. World Health Organization. Media Centre/ Diabetes (2006) Definition and diagnosis of diabetes mellitus and intermediate hyperglycaemia. [Internet]. Geneva: WHO [cited 2014 Marc 30]. Available from: http://whqlibdoc.who. int/publications/2006/9241594934_eng.pdf.

23. Ainamo J, Bay I. Problems and proposals for recording gingivitis and plaque. Int Dent J. 1975 Dec;25(4):229-35.

24. Kardeşler L, Buduneli N, Cetinkalp S, Kinane DF. Adipokines and inflammatory mediators after initial periodontal treatment in patients with type 2 diabetes and chronic periodontitis. J Periodontol. 2010 Jan;81(1):24-33.

25. McNabb H, Mombelli A, Gmür R, Mathey-Dinç S, Lang NP. Periodontal pathogens in the shallow pockets of immigrants from developing countries. Oral Microbiol Immunol. 1992 Oct;7(5):267-72.

26. Horliana AC, Chambrone L, Foz AM, Artese HP, Rabelo MS, Pannuti CM, et al. Dissemination of periodontal pathogens in the bloodstream after periodontal procedures: a systematic review. PloS One. 2014 May 28;9(5):e98271.

27. Hujoel PP, Drangsholt M, Spiekerman C, Derouen TA. Examining the link between coronary heart disease and the elimination of chronic dental infections. J Am Dent Assoc. 2001 Jul;132(7):883-9.

28. Loos BG, Craandijk J, Hoek FJ, Wertheim-van Dillen PM, van der Velden U. Elevation of systemic markers related to cardiovascular diseases in the peripheral blood of periodontitis patients. J Periodontol. 2000 Oct;71(10):1528-34.

29. Li J, Guo Y, Luan X, Qi T, Li D, Chen Y, et al. Independent roles of monocyte chemoattractant protein-1, regulated on activation, normal T-cell expressed and secreted and fractalkine in the vulnerability of coronary atherosclerotic plaques. Circ J. 2012;76(9):2167-73. 